\title{
Development of patent Litomosoides sigmodontis infections in semi-susceptible C57BL/6 mice in the absence of adaptive immune responses
}

\author{
Laura E. Layland*, Jesuthas Ajendra, Manuel Ritter, Anna Wiszniewsky, Achim Hoerauf and Marc P. Hübner
}

\begin{abstract}
Background: One of the most advantageous research aspects of the murine model of filariasis, Litomosoides sigmodontis, is the availability of mouse strains with varying susceptibility to the nematode infection. In C57BL/6 mice, L. sigmodontis worms are largely eliminated in this strain by day 40 post-infection and never produce their offspring, microfilariae (Mf). This provides a unique opportunity to decipher potential immune pathways that are required by filariae to achieve a successful infection. In this study we tracked worm development and patency, the production of microfilariae and thus the transmission life-stage, in Rag2IL-2R $\gamma^{-1-}$ mice which are deficient in T, B and NK cell populations.
\end{abstract}

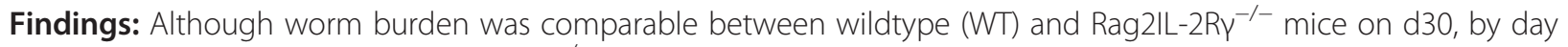
72 post-infection, parasites in Rag2IL-2R $\gamma^{-1-}$ mice were still in abundance, freely motile and all mice presented high quantities of Mf both at the site of infection, the thoracic cavity (TC), and in peripheral blood. Levels of cytokine (IL-4, IL-6, TNFa) and chemokine (MIP-2, RANTES, Eotaxin) parameters were generally low in the TC of infected

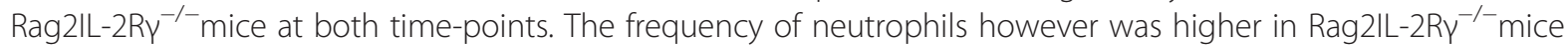
whereas eosinophils and macrophage populations, including alternatively activated macrophages, were elevated in WT controls.

Conclusion: Our data highlight that adaptive immune responses prevent the development of patent L. sigmodontis infections in semi-susceptible C57BL/6 mice and suggest that induction of such responses may offer a strategy to prevent transmission of human filariasis.

Keywords: Litomosoides sigmodontis, Filariae, C57BL/6, Patency, Immune-regulation

\section{Background}

Filariasis remains a major cause of severe morbidity and socioeconomic difficulties in the tropics [1]. Infections persist for numerous years in man due to regulatory strategies developed by the nematode [2]. To understand the principle immune players which govern worm burden and control pathology, researchers use rodent models of the disease such as Litomosoides sigmodontis [3]. In BALB/c mice, this model allows the comparison of developing immune responses against all stages of the

\footnotetext{
* Correspondence: laura.layland@microbiology-bonn.de

Institute of Medical Microbiology, Immunology and Parasitology (IMMIP),

University Hospital of Bonn, Sigmund Freud Straße 25, Bonn 53105, Germany
}

helminth's life-cycle, including the release of Mf, the worm's offspring [4]. C57BL/6 mice on the other hand are refractory to full development of the parasite and progressively eliminate adult worms 40 days post-infection (p.i.) $[5,6]$. In all hosts, penetrating $L$. sigmodontis larvae migrate through the lymphatics and settle in the TC after a few days. These larvae then moult into L4 ( +9 days p.i.) and then again into adults ( +28 days p.i.). During infection, granuloma-like structures encase the filariae but the composition of granulocytes and macrophages within the granulomas does not precisely coincide with the cellular infiltration into the TC [7]. This study followed filarial development in B6-Rag2 ${ }^{\mathrm{tm} 1 \mathrm{Fwa}} \mathrm{II} 2 \mathrm{rg}^{\mathrm{tm} 1 \mathrm{Wjl}}$ C57BL/6 mice 
which are deficient in T, B and NK cell populations $[8,9]$. L. sigmodontis infections in these mice transpired into full patency with long free-living worms and no encapsulation was observed at either $\mathrm{d} 30$ or $\mathrm{d} 72$ of infection. In addition, whereas the frequencies of eosinophils and RELM $\alpha$-positive AAM (alternatively activated macrophage) populations were elevated in WT mice in the TC, neutrophil populations were most prominent in Rag2IL-2R $\gamma^{-/-}$C57BL/6 mice. Although previous studies have indicated that parasite clearance in non-susceptible mice is Th2 dependent [10], our new findings indicate that other immune factors are also required such as NK cell populations.

\section{Findings \\ Methods}

Animals, ethics and infection

B6-Rag2 ${ }^{\text {tm1Fwa }} I I 2$ rg $^{\text {tm1Wjl }}$ mice (http://www.taconic.com/ 4111) were purchased from Taconic Biosciences Inc, Cologne, Germany and bred alongside WT C57BL/6 mice. Mice were kept under SPF conditions and experiments were in accordance with local government authorities (No.87-51.04.2011.A025/01). Infections and parasite recovery with $L$. sigmodontis were performed as previously described [11]. Levels of Wolbachia DNA were performed using a duplex PCR measuring Wolbachia FtsZ and Actin [12].

\section{Flow cytometry staining of TC cells}

Isolated TC cells were prepared for flow cytometry as previously described [11]. In short, fixed cells were stained with SiglecF-PE (BD Bioscience, Heidelberg, Germany), F4/80-PerCP-Cy5.5, Gr1-PE-Cy7, CD86-FITC and MHC II-APC (all eBioscience, San Diego, USA). AAM were identified using intracellular staining for RELM $\alpha$ with rabbit anti-mouse RELM $\alpha$ (Peprotech, Rocky Hill, USA) followed by goat anti-rabbit Alexa Fluor 488-conjugated antibody (Invitrogen, Carlsbad, USA). Gating strategies are shown in Additional file 1. Flow cytometry was performed using a BD FACS Canto I and analyzed using FACSDiva 5.2 software (BD Biosciences).

\section{Cytokine determination}

Cytokine concentrations in the TC fluid were measured using ELISA in accordance to the manufacturer's instructions (IL-6, RANTES, TNF- $\alpha$, MIP-2 and Eotaxin all from RnD Systems, Minneapolis USA; IL-4 from BD Biosciences). ELISA plates were read and analyzed at 450 and $540 \mathrm{~nm}$ (Molecular Devices, Sunnyvale, CA, USA).

\section{Statistical analysis}

Statistical differences were determined using GraphPad Prism 5 software (San Diego, CA, USA). Parametrically distributed data were analyzed using unpaired t-tests or one-way ANOVA whereas non-parametrically distributed data were calculated using Kruskal-Wallis-tests. If significant, this was followed by a Mann-Whitney-U test for a further comparison of the groups.

\section{Results \& discussion}

Patent $L$. sigmodontis infection in Rag2IL-2R $\gamma$-deficient C57BL/6 mice

Male Rag2IL-2R ${ }^{-1-}$ and WT C57BL/6 mice were naturally infected with L. sigmodontis. After 30 days, mice were assessed for worm burden (Fig. 1a) and stage (Additional file 2A) since this time-point corresponds with the final moulting phase from L4 into adulthood. Although no significant differences in total worm burden were observed on d30 (Fig. 1a), Rag2IL-2R $\gamma^{-1-}$ mice had significantly higher numbers of adult worms compared to L4 (Additional file 2A) indicating an earlier moult into adulthood. On day 72, worms in all WT mice had been encapsulated into granulomatous nodules that were absence in Rag2IL- $2 \mathrm{R}^{-1-}$ mice. Filariae in Rag2IL-2R ${ }^{-1-}$ mice however were freely motile and in abundance (Fig. 1a). Both adult worm genders in Rag2IL-2R ${ }^{-1-}$ mice were also significantly longer in length and substantially grew between $\mathrm{d} 30$ and 72 (Fig. 1b and Additional file 2B). Levels of Wolbachia DNA in adult female worms were also significantly higher than in females from control mice (Fig. 1c). Their healthy state was further reflected in the production of Mf (Fig. 1d and Additional file 2C). Indeed, from day 49 p.i., increasing numbers of $\mathrm{Mf}$ could be detected in the periphery of all infected Rag2IL-2 $\mathrm{R}^{-1-}$ mice $(\mathrm{n}=16$ in three independent infection experiments) whereas none occurred in WT mice (Fig. 1d and Additional file 2C). Fig. 1e also shows correspondingly high levels of $\mathrm{Mf}$ in the TC of $\mathrm{KO}$ mice on the day of analysis (d72) and Mf length was $91.2 \pm 6.1 \mu \mathrm{m}$. Previous studies have demonstrated the development of $L$. sigmodontis patency in IL- $4^{-/-}$C57BL/6 mice. One study demonstrated that a s.c. injection of L3 into IL-4-deficient C57BL/6 mice resulted in a patent state in $33 \%$ of $L$. sigmodontis-infected mice [10]. Further studies have shown that IL-10 counter-regulates these effects since IL-4/IL-10 $0^{-/-}$mice failed to develop a patent state [13]. Another study using RAG2/IL-4-deficient mice reported significantly higher microfilariae levels than in susceptible $\mathrm{BALB} / \mathrm{c}$ mice, although the prevalence of patency was not discussed [14]. Our study highlights therefore, that lack of T, B and NK cells suffices to render C57BL/6 mice susceptible, leading to a $100 \%$ prevalence of patent animals that exceeds the impact of a single IL-4-deficient mice. Since L. sigmodontis-infected $\mu \mathrm{MT}$ C57BL/6 mice do not develop patent infections it appears that a lack of $B$ cells does not facilitate the development of patency [10]. Nevertheless, the importance of B and T cells was 


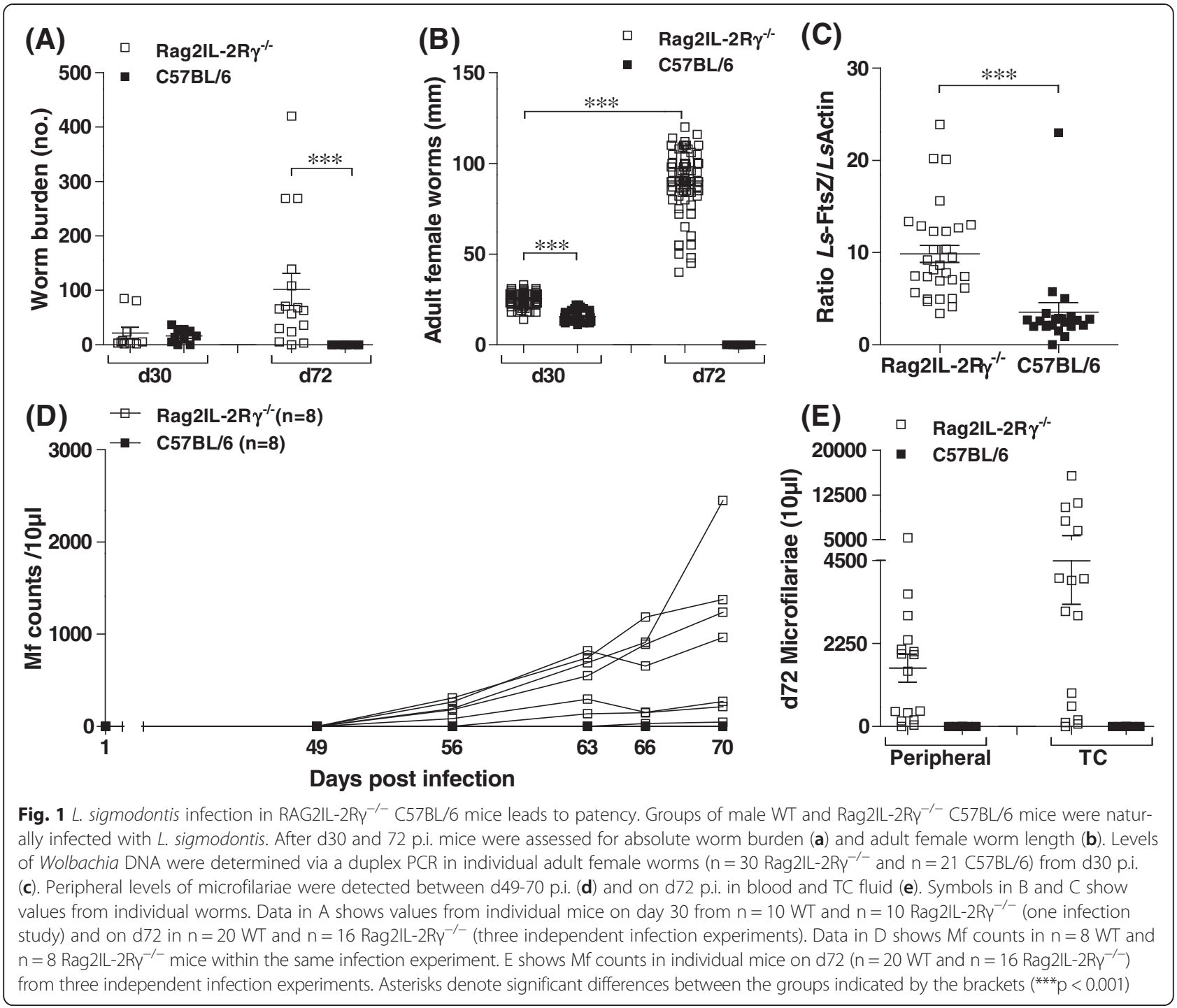

previously demonstrated in infections with $L$. sigmodontis susceptible BALB/c mice, since B1 cell-deficient (Xid) and $\mathrm{CD}^{+} \mathrm{T}$ cell depleted mice had increased worm burden and microfilariae $[15,16]$. However, lack of IL-4 in BALB/ c mice also increased patency but not worm load [17] indicating that IL-4-mediated immune responses hinder Mf release but not necessarily worm development.

\section{Elevated levels of MIP-2 in the TC of C57BL/6 mice upon elimination of $L$. sigmodontis filariae}

As IL-4 is critical for sexual maturity and Mf release in C57BL/6 mice $[10,13]$, we determined IL-4 levels in the $\mathrm{TC}$ on $\mathrm{d} 30$ and 72 in infected groups. Whereas no IL-4 could be detected in Rag2IL-2R $\gamma^{-/-}$mice at either timepoint, levels in WT mice were significantly elevated on $\mathrm{d} 30$ when compared to the Rag2IL-2R $\gamma^{-/-}$group and WT mice on d72 (Fig. 2a). Based on the differences in frequency of patent animals between IL-4 deficient and
Rag2IL-2 $\mathrm{R}^{-/-}$mice, we assume that the lack of $\mathrm{T}$ and $B$ cells exceeds the importance of IL-4 alone in Rag2IL-2 $\mathrm{R}^{-/-}$mice accounting for the observed phenotype. Interestingly levels of another Th2 cytokine, IL-13, were previously shown to be comparable in $L$. sigmodontis-infected WT and IL- $4^{-/-}$mice [13]. In the study here, levels of IL- 6 in the TC of WT mice were also significantly higher on d30 than d72 (Fig. 2b) but levels of TNF- $\alpha$ in WT mice were only significantly elevated on $\mathrm{d} 72$ p.i. when compared to Rag2IL- $2 \mathrm{R}^{-/-}$mice (Fig. 2c). This pattern was also observed with levels of MIP2 and interestingly, levels of this chemokine were also significantly elevated in WT mice on $\mathrm{d} 72$ when compared to WT mice on d30 (Fig. 2d). Levels of RANTES in the TC, reflected measurements of IL-4, that is, significantly higher amounts on day 30 when compared to d72 (Fig. 2e). Levels of Eotaxin-1 however were very low in all groups at both time-points (Fig. 2f). TC levels of these parameters 

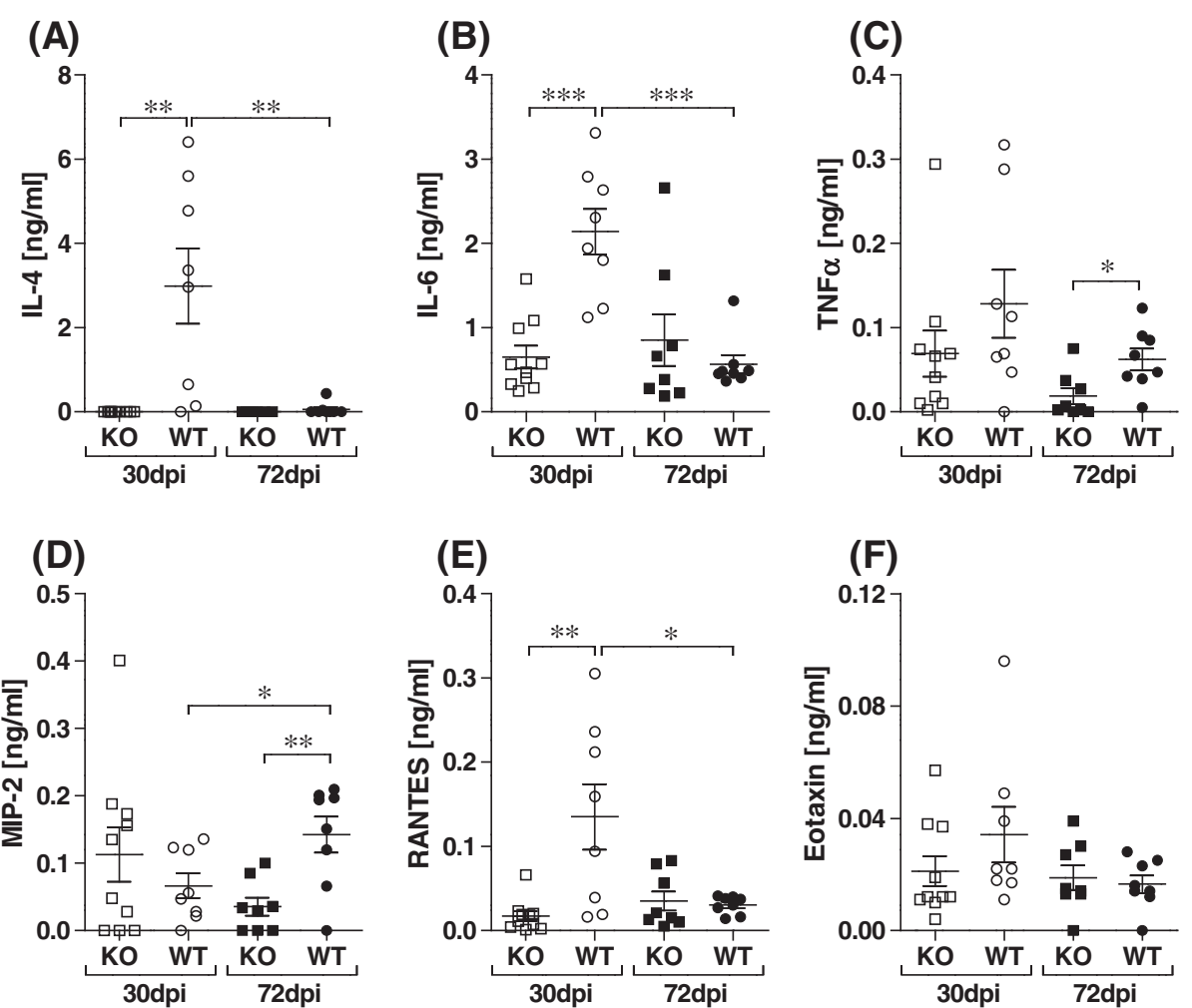

Fig. 2 Low levels of cytokine and chemokines in the TC of L. sigmodontis-infected Rag2IL-2RY ${ }^{-1-}$ C57BL/6 mice. Levels of IL-4 (a), IL-6 (b), TNF-a (c), MIP-2 (d), RANTES (e) and Eotaxin-1 (f) were measured in the TC fluid from individual mice on days 30 and 72 p.i. by ELISA. Symbols show levels in individual mice. Graphs show data from one infection experiment comprising of $n=10 \mathrm{WT}$ and $n=10$ Rag2lL-2R $\gamma^{-/-}$on $\mathrm{d} 30$ and $n=8 \mathrm{WT}$ and $n=8$ Rag2IL-2RY ${ }^{-1-}$ on d72. Asterisks denote significant differences $n=10$ between the groups indicated by the brackets $\left({ }^{*} p<0.01,{ }^{* *} p<0.05,{ }^{* * *} p<0.001\right)$

were analysed in two further independent infection experiments on $\mathrm{d} 72(\mathrm{n}=10$ WT and $\mathrm{n}=8$ Rag2IL$2 \mathrm{R}^{-/-}$mice) and levels were comparable to those depicted in Fig. 2 (data not shown). Those results are in accordance to the well described role of $\mathrm{T}$ and $\mathrm{B}$ cells to drive immune responses.

\section{L. sigmodontis-infected Rag2IL-2R ${ }^{-/-}$C57BL/6 mice present elevated neutrophils but reduced AAM populations at the site of infection}

Next we studied changes in immune cell populations in the TC. At both analysed time-points, SiglecF $\mathrm{F}^{+} \mathrm{F} / 80^{-}$ eosinophil populations remained significantly elevated in WT mice (Fig. 3a), whereas $\mathrm{Gr}^{+} \mathrm{SiglecF}^{-} \mathrm{F} 4 / 80^{-}$neutrophils were higher in Rag2IL-2 $\mathrm{R}^{-/-}$mice (Fig. 3b). The role of neutrophils and eosinophils in controlling filarial development and granuloma development has been well studied [18-20]. The elevated frequency of neutrophils in Rag2IL-2R ${ }^{-1-}$ mice here suggests that neutrophils require further signals to develop granulomas and/or control worm burden. Since Rag2IL-2R ${ }^{-1-}$ mice also lack NK populations it will be interesting to observe the effects on worm burden in these mice upon reconstitution of IL-4 competent NK cells. The actual role of NK cells in filarial infections is not well-defined although studies with $L$. sigmodontis, have shown that depletion of NK cells enhanced worm load and Th2 responses at the site of infection [21]. This correlates to the findings observed here with Rag2IL-2R ${ }^{-1-}$ mice since they also lack NK cell populations. With regards to macrophage populations, the percentage of $\mathrm{F} 4 / 80^{\mathrm{hi}}$ cells was significantly higher in WT mice on d72 of infection (Fig. 3c). However, MHCII and CD86 expressing F4/80 hi populations were elevated in Rag2IL-2R $\gamma^{-/-}$mice on d72 p.i., highlighting an increased activation (Figs. $3 \mathrm{~d}$ and e respectively). Finally, we also determined the frequency of AAM population using RELM- $\alpha$, at both time-points, this population was significantly higher in WT mice (Fig. 3f). Previous studies have elucidated that in the absence of recruitment signalling, IL-4 drives macrophage proliferation and reduces the development of AAM populations [22]. Interestingly, acute infected $L$. sigmodontis $\operatorname{Rag} 1^{-1-}$ C57BL/6 mice showed no development of $\mathrm{Ym}^{+}$(AAM) [22] which correlates to the 


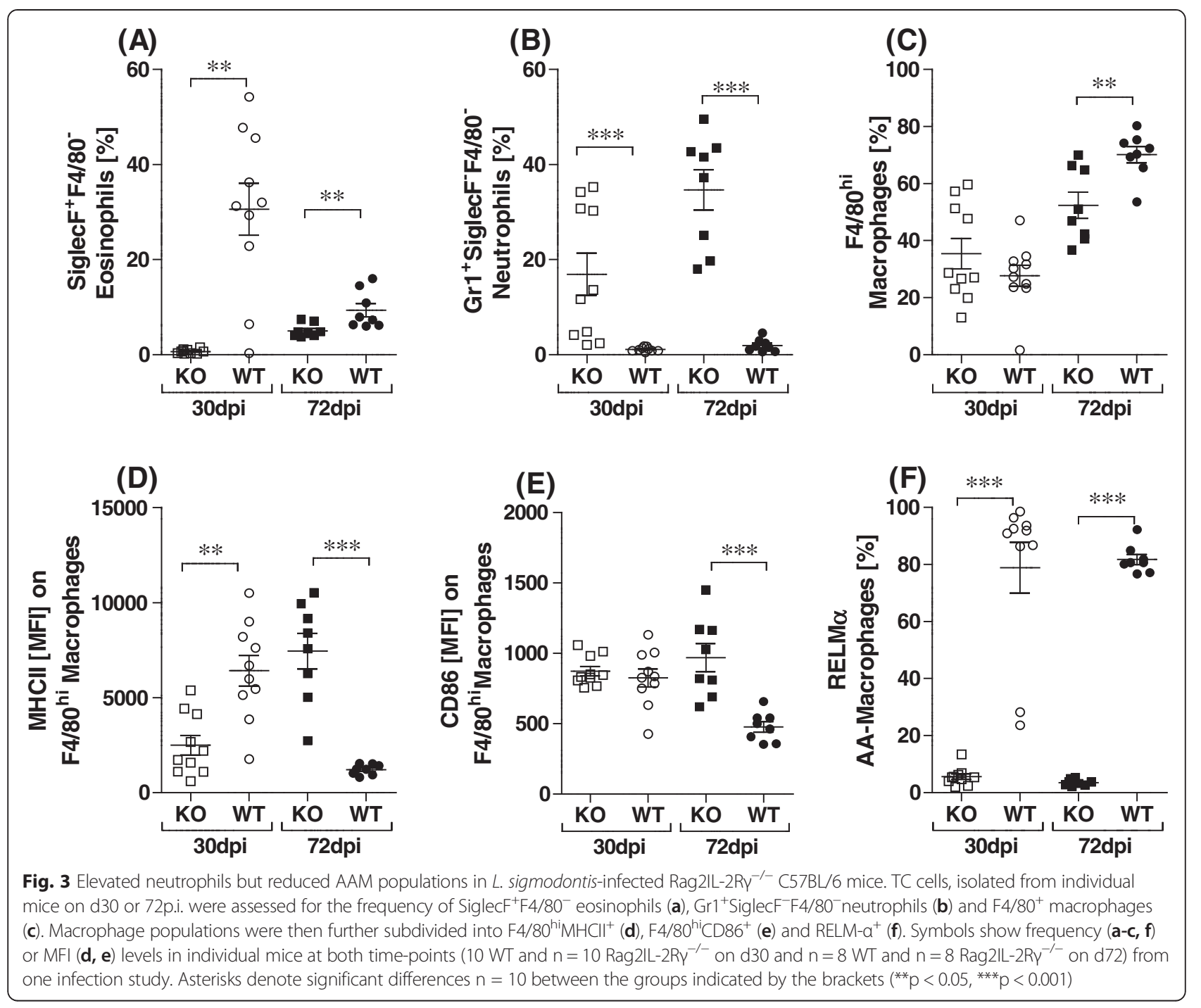

lack of AAM in the Rag2IL- $2 \mathrm{R}^{-1-}$ mice studied here, demonstrating that adaptive immune responses appear fundamental for AAM development. Flow cytometry data in two further independent infection experiments analysed on $\mathrm{d} 72\left(\mathrm{n}=10 \mathrm{WT}\right.$ and $\mathrm{n}=8 \mathrm{Rag} 2 \mathrm{IL}-2 \mathrm{R} \gamma^{-/-}$ mice) were comparable (data not shown). Future studies will have to analyze the impact of specific cell populations including AAM, T cell subsets, B cells and NK cells using Rag-deficient strains to further elucidate mechanisms that could be targeted to prevent the development of patent infections.

\section{Conclusion}

In the current study we demonstrate that deficiency in $\mathrm{T}, \mathrm{B}$, and NK cells renders semi-susceptible C57BL/6 mice to $100 \%$ patent during L. sigmodontis infection. Using the L. sigmodontis Rag2IL-2R $\gamma^{-/-}$model offers therefore the opportunity to identify cell types that are involved in the development of patency, presenting new targets to combat filarial transmission.

\section{Additional files}

Additional file 1: Gating strategies for flow cytometry. Groups of male WT and Rag2IL-2RY ${ }^{-1-}$ C57BL/6 mice were infected with L. sigmodontis. After 72 days of infection, cells from the TC were isolated and screened for different cell populations by flow cytometry. A-C: Gating Strategy for TC cells, gates for macrophages, eosinophils, neutrophils. D: Gating strategy for AAM and E: Plots for expression of CD86 and MHCII in macrophages. (PPT $142 \mathrm{~kb}$ )

Additional file 2: Infections with L. sigmodontis in Rag2IL-2R $\gamma^{-1-}$ C57BL/ 6 mice drives faster moulting into adulthood. Groups of male WT and Rag2IL-2RY ${ }^{-1-}$ C57BL/6 mice were infected with L. sigmodontis. After d30 p.i. worms were assessed for larval or adult life-stages (A). Symbols show the number of different worm stages recovered from individual mice $(n=10 \mathrm{WT}$ and $\mathrm{n}=10$ Rag2 $\mid \mathrm{L}-2 \mathrm{R} \mathrm{Y}^{-1-}$ ). (B), on d30 and d72, adult male worm length was 
determined. (C) Peripheral levels of microfilariae were determined from d49 until $d 70$ p.i. Each symbol represents the Mf load in individual mice $(n=10$ $\mathrm{WT}$ and $\mathrm{n}=8$ Rag2 $\mathrm{LL}-2 \mathrm{RY}^{-1-}$ ) from two independent infection experiments. Asterisks denote significant differences between the groups indicated by the brackets $\left({ }^{* * *} \mathrm{p}<0.001\right)$. (PPTX $\left.63 \mathrm{~kb}\right)$

\section{Abbreviations}

L. sigmodontis: Litomosoides sigmodontis; Mf: Microfilariae; TC: Thoracic cavity.

\section{Competing interests}

The authors declare that they have no competing interests.

\section{Authors' contributions}

LEL and MPH designed the study. JA, MR, AW performed the experiments. LEL and JA analysed the data. LEL wrote the manuscript which was then revised by MPH and AH and further approved by all other authors. All authors read and approved the final manuscript.

\section{Authors' information}

Achim Hoerauf and Marc P. Hübner shared authorship.

\section{Acknowledgements}

Special thanks to Ö. Mutluer, V. Krupp and K. Wiszniewsky (IMMIP) for excellent technical assistance. This work was primarily supported through a grant from the German Research Council (DFG HU 2144/1-1). The study was further supported by the BONFOR intramural funding program of the Medical Faculty of Bonn University and the European Commission: Enhanced Protective Immunity Against Filariasis (EPIAF), agreement number 242131. LEL and AH are recipients of further DFG funding within the "African-German Cooperation Projects in Infectiology" (HO 2009/10-1). AH is a member of the Excellence Cluster Immunosensation (DFG, EXC 1023) and of the German Centre of Infectious Disease (DZIF).

\section{Received: 5 December 2014 Accepted: 16 July 2015}

Published online: 25 July 2015

\section{References}

1. World Health Organization. African Programme for Onchocerciasis Control: meeting of national onchocerciasis task forces. Weekly epidemiological record September. 2012:87:493-508.

2. Metenou S, Nutman TB. Regulatory T Cell Subsets in Filarial Infection and Their Function. Front Immunol. 2013;4:305

3. Morris CP, Evans H, Larsen SE, Mitre E. A comprehensive, model based review of vaccine and repeat infection trials for filarasis. Clin Micrbiol Rev. 2013;26:381-421.

4. Petit G, Diagne M, Marechal P, Owen D, Taylor D, Bain O. Maturation of the filaria Litomosoides sigmodontis in BALB/c mice; comparative susceptibility of nine other inbred strains. Ann Parasitol Hum Comp. 1992;67:144-50.

5. Hoffmann W, Petit G, Schulz-Key H, Taylor D, Bain O, Le Goff L. Litomosoides sigmodontis in mice: reappraisal of an old model for filarial research. Parasitol Today. 2000;16:387-9.

6. Babayan S, Ungeheuer MN, Martin C, Attout T, Belnoue E, Snounou G, et al. Resistance and susceptibility to filarial infection with Litomosoides sigmodontis are associated with early differences in parasite development and in localized immune reactions. Infect Immun. 2003;71:6820-9.

7. Attout T, Coralie MC, Babayan SA, Kozek WJ, Bazzocchi C, Oudet F, et al. Pleural cellular reaction to the filarial infection Litomosoides sigmodontis is determined by the moulting process, the worm alteration, and the host strain. Parasitol International. 2008:57:201-11.

8. Shinkai Y, Rathbun G, Lam KP, Oltz EM, Stewart V, Mendelsohn M, et al. RAG-2-deficient mice lack mature lymphocytes owing to inability to initiate V(D)J rearrangement. Cell. 1992;6:855-67.

9. Cao X, Shores EW, Hu-Li J, Anver MR, Keisall BL, Russell SM, et al. Defective lymphoid development in mice lacking expression of the common cytokine receptor $\gamma$ chain. Immunity. 1995;2:223-38.

10. Le Goff L, Lamb TJ, Graham AL, Harcus Y, Allen JE. IL-4 is required to prevent filarial nematode development in resistant but not susceptible strains of mice. Int J Parasitol. 2002;32:1277-84.

11. Ajendra J, Specht S, Neumann AL, Gondorf F, Schmidt D, Gentil K, et al. ST2 deficiency does not impair type 2 immune responses during chronic filarial infection but leads to an increased microfilaremia due to an impaired splenic microfilarial clearance. PLoS One. 2014;3:e93072.

12. Schiefer A, Schmitz A, Schäberle TF, Specht S, Lämmer C, Johnston KL, et al. Corallopyronin A specifically targets and depletes essential obligate Wolbachia endobacteria from filarial nematodes in vivo. J Infect Dis. 2012;206:249-57.

13. Specht S, Volkmann L, Wynn T, Hoerauf A. Interleukin-10 (IL-10) counterregulates IL-4-dependent effector mechanisms in Murine Filariasis. Infect Immun. 2004;72:6287-93.

14. Babayan SA, Read AF, Lawrence RA, Bain O, Allen JE. Filarial parasites develop faster and reproduce earlier in response to host immune effectors that determine filarial life expectancy. PLoS Biol. 2010;8:e1000525.

15. Al-Qaoud KM, Fleischer B, Hoerauf A. The Xid defect imparts susceptibility to experimental murine filariosis-association with a lack of antibody and IL-10 production by B cells in response to phosphorylcholine. Int Immunol. 1998;10:17-25.

16. Al-Qaoud KM, Taubert A, Zahner H, Fleischer B, Hoerauf A. Infection of BALB/C mice with the filarial nematode Litomosoides sigmodontis: role of CD4+ T cells in controlling larval development. Infect Immun. 1997;65:2457-61.

17. Volkmann L, Saeftel M, Bain O, Fischer K, Fleischer B, Hoerauf A. Interleukin-4 is essential for the control of microfilariae in murine infection with the filaria Litomosoides sigmodontis. Infect Immun. 2001;69:2950-6.

18. Brattig NW, Büttner DW, Hoerauf A. Neutrophil accumulation around Onchocerca worms and chemotaxis of neutrophils are dependent on Wolbachia endobacteria. Microbes Infect. 2001;6:439-46.

19. Saeftel M, Volkmann L, Korten S, Brattig N, Al-Qaoud K, Fleischer B, et al. Lack of interferon-gamma confers impaired neutrophil granulocyte function and imparts prolonged survival of adult filarial worms in murine filariasis. Microbes Infect. 2001;3:203-13.

20. Al-Qaoud KM, Pearlman E, Hartung T, Klukowski J, Fleischer B, Hoerauf A. A new mechanism for IL-5-dependent helminth control: neutrophil accumulation and neutrophil-mediated worm encapsulation in murine filariasis are abolished in the absence of IL-5. Int Immunol. 2000;12:899-908.

21. Korten S, Volkmann L, Saeftel M, Fischer K, Taniguchi M, Fleischer B, et al. Expansion of NK cells with reduction of their inhibitory Ly-49A, Ly-49C, and Ly-49G2 receptor-expressing subsets in a murine helminth infection: contribution to parasite control. J Immunol. 2002;168:5199-206.

22. Jenkins SJ, Ruckerl D, Thomas GD, Hewitson JP, Duncan S, Brombacher F, et al. IL-4 directly signals tissue-resident macrophages to proliferate beyond homeostatic levels controlled by CSF-1. J Exp Med. 2013;210:2477-91.

\section{Submit your next manuscript to BioMed Central and take full advantage of:}

- Convenient online submission

- Thorough peer review

- No space constraints or color figure charges

- Immediate publication on acceptance

- Inclusion in PubMed, CAS, Scopus and Google Scholar

- Research which is freely available for redistribution 